\title{
The Role of Consolidation Chemoradiotherapy in Locally Advanced Pancreatic Cancer Receiving Chemotherapy: An Updated Systematic Review and Meta-Analysis
}

\author{
Jeffrey S. Chang, MD, $P h D^{1}$ \\ Yen-Feng Chiu, $\mathrm{PhD}^{2}$ \\ Jih-Chang $\mathbf{Y u}, \mathrm{PhD}^{2}$ \\ Li-Tzong Chen, MD, PhD',3,4 \\ Hui-Ju Ch'ang, MD ${ }^{1,5,6}$
}

\begin{abstract}
${ }^{1}$ National Institute of Cancer Research, ${ }^{2}$ Institute of Population Health Sciences, National Health Research Institutes, Zhunan, ${ }^{3}$ Department of Internal Medicine, National Cheng Kung University Hospital, Tainan, ${ }^{4}$ Department of Internal Medicine, Kaohsiung Medical University Hospital, Kaohsiung Medical University, Kaohsiung, ${ }^{5}$ Program for Cancer Biology and

Drug Discovery, College of Medical Science and Technology, Taipei Medical University, Taipei, ${ }^{6}$ Taipei Cancer Center,

Taipei Medical University, Taipei, Taiwan
\end{abstract}

\begin{abstract}
Purpose
The role of consolidation chemoradiation (CCRT) after systemic chemotherapy in locally advanced pancreatic cancer (LAPC) is still controversial. We aim to evaluate the effectiveness of CCRT in LAPC using systematic review and meta-analysis of prospective studies.
\end{abstract}

\section{Materials and Methods}

Prospective clinical trials of LAPC receiving chemotherapy with or without subsequent CCRT were included in the analysis. We systematically searched in PubMed, MEDLINE, Embase, and Web of Science. The primary outcome of interest was 1-year survival. Secondary endpoints were median overall survival, progression-free survival, toxicity, and resection rate.

\section{Results}

Forty-one studies with 49 study arms were included with a total of 1,018 patients receiving CCRT after induction chemotherapy (ICT) and 954 patients receiving chemotherapy alone. CCRT after ICT did not improve 1-year survival significantly in LAPC patients compared with chemotherapy alone (58\% vs. 52\%). ICT lasted for at least 3 months revealed significantly improved survival of additional CCRT to LAPC patients compared to chemotherapy alone (65\% vs. 52\%). A marginal survival benefit of consolidation CCRT was noted in studies using maintenance chemotherapy (59\% vs. 52\%), and fluorouracil-based CCRT (64\% vs. 52\%), as well as in studies conducted after the 2010 (64\% vs. 55\%).

\section{Conclusion}

The survival benefit of ICT+CCRT over chemotherapy alone in treating LAPC was noted when ICT lasted for at least 3 months. Fluorouracil-based CCRT, and maintenance chemotherapy were associated with improved clinical outcomes.
Correspondence: Hui-Ju Ch'ang, MD

National Institute of Cancer Research, National Health Research Institutes, R1-2034, 35 Keyan road, Zhunan, Miaoli County 35053, Taiwan Tel: 886-37-246-166 (ext. 35105)

Fax: 886-37-586-463

E-mail: hjmc@nhri.org.tw

Received March 2, 2017

Accepted May 31, 2017

Published Online June 9, 2017

\section{Key words}

Pancreatic neoplasms, Adenocarcinoma, Induction chemotherapy, Chemoradiotherapy

\section{Introduction}

Although most pancreatic cancers are diagnosed at advanced stage, around $50 \%$ of pancreatic cancer patients present with localized disease without evident distant metastasis. Locally advanced pancreatic cancers (LAPC) include a heterogenous spectrum of disease from borderline resectable to unresectable tumors [1]. Chemoradiotherapy was once the standard of care for LAPC [2]. With gemcitabine becoming the preferred treatment strategy for advanced pancreatic cancer [3], clinical trials comparing chemoradiotherapy with gemcitabine alone in LAPC patients showed mixed results $[4,5]$. Meta-analysis revealed similar clinical outcomes using up-front chemoradiotherapy followed by chemotherapy versus chemotherapy alone in LAPC [6].

The development of induction chemotherapy (ICT) followed sequentially by consolidation chemoradiotherapy (CCRT) was based on the rationale of sparing patients with rapidly progressive disease from radical local radiotherapy. 
Some retrospective studies suggested that this approach could improve survival of LAPC $[7,8]$. The only prospective randomized trial evaluating CCRT in addition to chemotherapy revealed an increase in progression-free survival (PFS), resulting in longer period without treatment (6.1 months vs. 3.7 months, $\mathrm{p}=0.02)$, and a decrease in local progression $(32 \%$ vs. $46 \%, \mathrm{p}=0.03$ ); while no survival benefit was observed [9]. However, the $68 \%$ minor to major radiation protocol violation in LAP07 study might underestimate the effect of chemoradiotherapy [10]. Another possibility contributing to the lack of overall survival (OS) benefit of CCRT might be the dominant effect of metastatic disease due to ineffective systemic chemotherapy.

Many prospective studies evaluating modern chemotherapy alone or ICT followed by CCRT in LAPC have been published, but the sample sizes of most studies were too small to draw definite conclusions. To evaluate the efficacy of CCRT after ICT and provide further information for the design of clinical trials, we conducted a systematic review and meta-analysis of prospective clinical trials that investigated the efficacy of chemotherapy alone or ICT followed by CCRT in treating LAPC.

\section{Materials and Methods}

A protocol was developed to collect data from prospective clinical trials for LAPC patients. The details were defined in a prospectively registered protocol available online (PROSPERO identifier CRD42016047182). The reporting of the current study adheres to the criteria included in the Preferred Reporting Items for Systemic Reviews and Meta-Analyses (PRISMA) [11].

Prospective clinical trials published in English were identified by searching PubMed, Embase, MEDLINE, Web of Science from the date of inception of each database to January 2016. The search included the following terms: "pancreatic cancer," "pancreatic carcinoma," "pancreatic adenocarcinoma," and "locally advanced," "unresectable," "non-metastatic." Additional clinical trials were identified by reviewing the previous meta-analyses.

\section{Eligibility criteria}

To be included in the meta-analysis, the study had to meet all the following criteria: (1) patients were locally advanced pancreatic ductal adenocarcinoma.; (2) prospective phase II or randomized trials; (3) treatment with chemotherapy alone or ICT followed by CCRT; (4) reported outcomes of interests, including 1-year survival rate and median OS (S1 Table).
When the eligibility criteria were met, the full article was then retrieved for further assessment. The discrepancies between two investigators were resolved by consensus after discussion.

\section{Data extraction and study endpoints}

Data were extracted independently by two investigators (H.-J.C. and J.S.C.) using a predefined data extraction sheet. The primary end-point was 1-year survival rate. Secondary end-points were PFS, median OS, toxicity, and resection rate.

\section{Statistical analysis}

The current meta-analysis on 1-year survival rate was performed with $\mathrm{R}$ function metaprop in R package meta [12] using $R$ statistical software ver. 3.2.2 (R Foundation for Statistical Computing, Vienna, Austria). Both the fixed-effects model and the random-effects model were used to perform meta-analysis. We applied Freeman-Tukey double arcsine transformation to 1-year survival rate to calculate the pooled estimates of proportions and their corresponding 95\% confidence intervals [13]. Heterogeneity between studies was evaluated by $\mathrm{I}^{2}$ statistic with $\mathrm{I}^{2}=0 \%-24.9 \%, 25 \%-49.9 \%$, and $\geq 50 \%$ representing low, moderate, and high heterogeneity, respectively [14]. The Cochran statistic was calculated to assess the significance level of $\mathrm{I}^{2}$ statistic [15]. A p-value of $<0.05$ indicated a significant heterogeneity between studies, and results generated by the random-effects models were reported. Results from the fixed-effects models were included when the homogeneity assumption was not violated $(p>0.05)$. Forest plot was used to display the probabilities of individual studies. The analysis was first performed to assess the overall difference in 1-year survival rate between the two treatment groups. Additional analyses were performed by study year, randomization, resectability status, number of chemotherapy agents, ICT duration, maintenance chemotherapy, radiotherapy dosage, and radiosensitizer. Statistical significance was determined by examining the 95\% confidence intervals (CIs). The comparison between chemotherapy versus ICT+CCRT was deemed statistically significant when the 95\% CIs did not overlap. Funnel plot and Egger's regression asymmetry test were used to evaluate publication bias [16,17].

\section{Results}

Fig. 1 presents the flow chart depicting the study selection process. After screening the titles or abstracts of 2,659 studies 


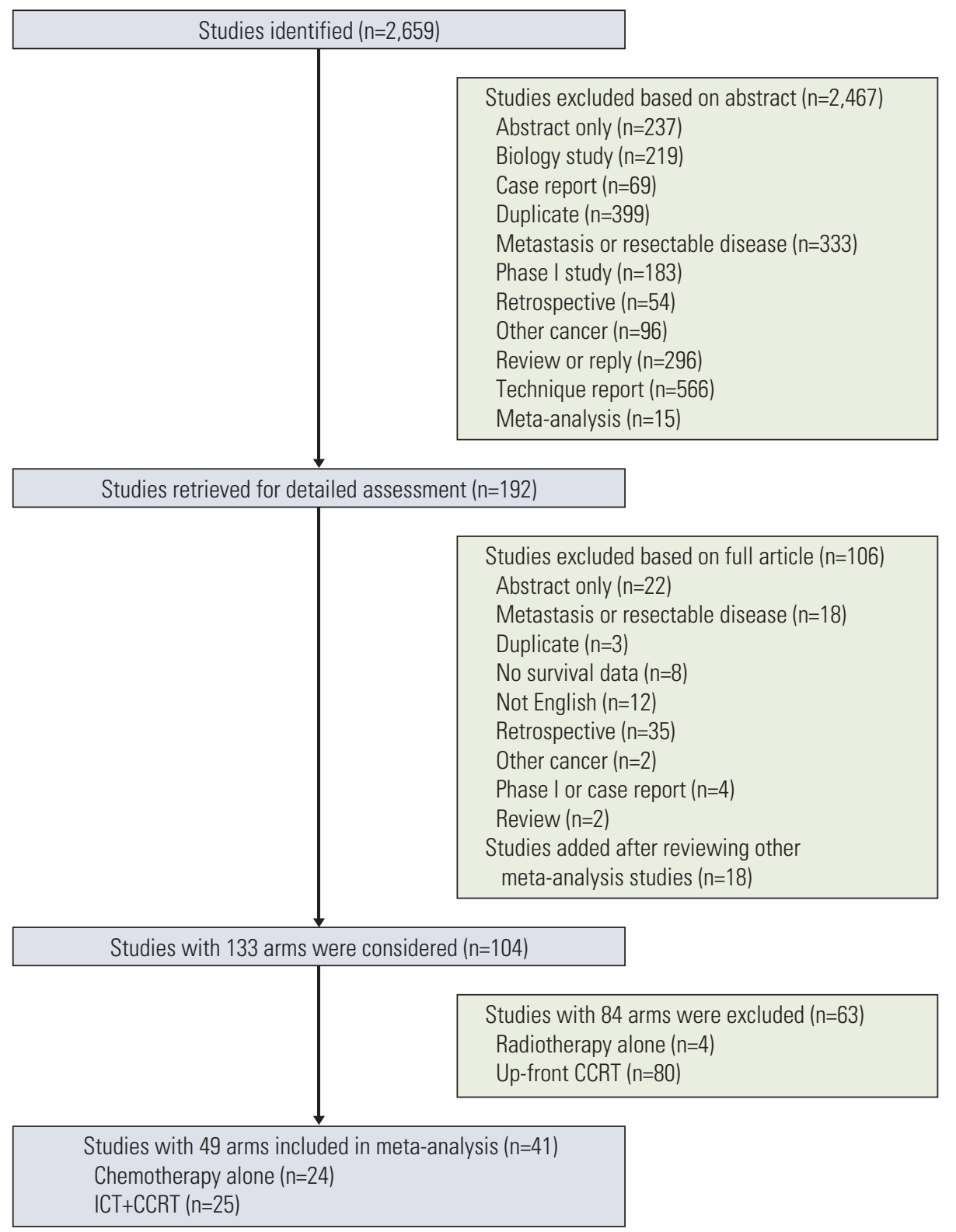

Fig. 1. Flow chart of study selection. CCRT, chemoradiation; ICT, induction chemotherapy.

identified using key words described in "Materials and Methods" (S2 Table), 192 studies were selected for full-text assessment, of which 106 studies were excluded due to retrospective analysis or studies enrolling metastatic/ resectable patients, etc. (S3 Table). Another 18 studies were added after reviewing other meta-analyses of LAPC treatment. Of the 110 studies with 134 study arms, 85 arms were excluded due to studies using radiotherapy alone or upfront chemoradiotherapy (S4 Table). The 41 studies in the final meta-analysis included 12 randomized studies and 29 phase II studies (S5
Table). The quality of each trial was evaluated (S6-S9 Tables). Of the 49 study arms, 24 arms were chemotherapy alone (Table 1) [18-35] and 25 arms were ICT followed by CCRT (Table 2) [35-58].

A total of 1,018 patients received ICT+CCRT, and 954 patients received chemotherapy alone. Twelve $(50 \%)$ and six (24\%) study arms of chemotherapy alone and ICT+CCRT, respectively, administered single chemotherapy agent. Twelve and eight studies used 5-fluorouracil and gemcitabine, respectively, as radiosensitizer. Two studies using 


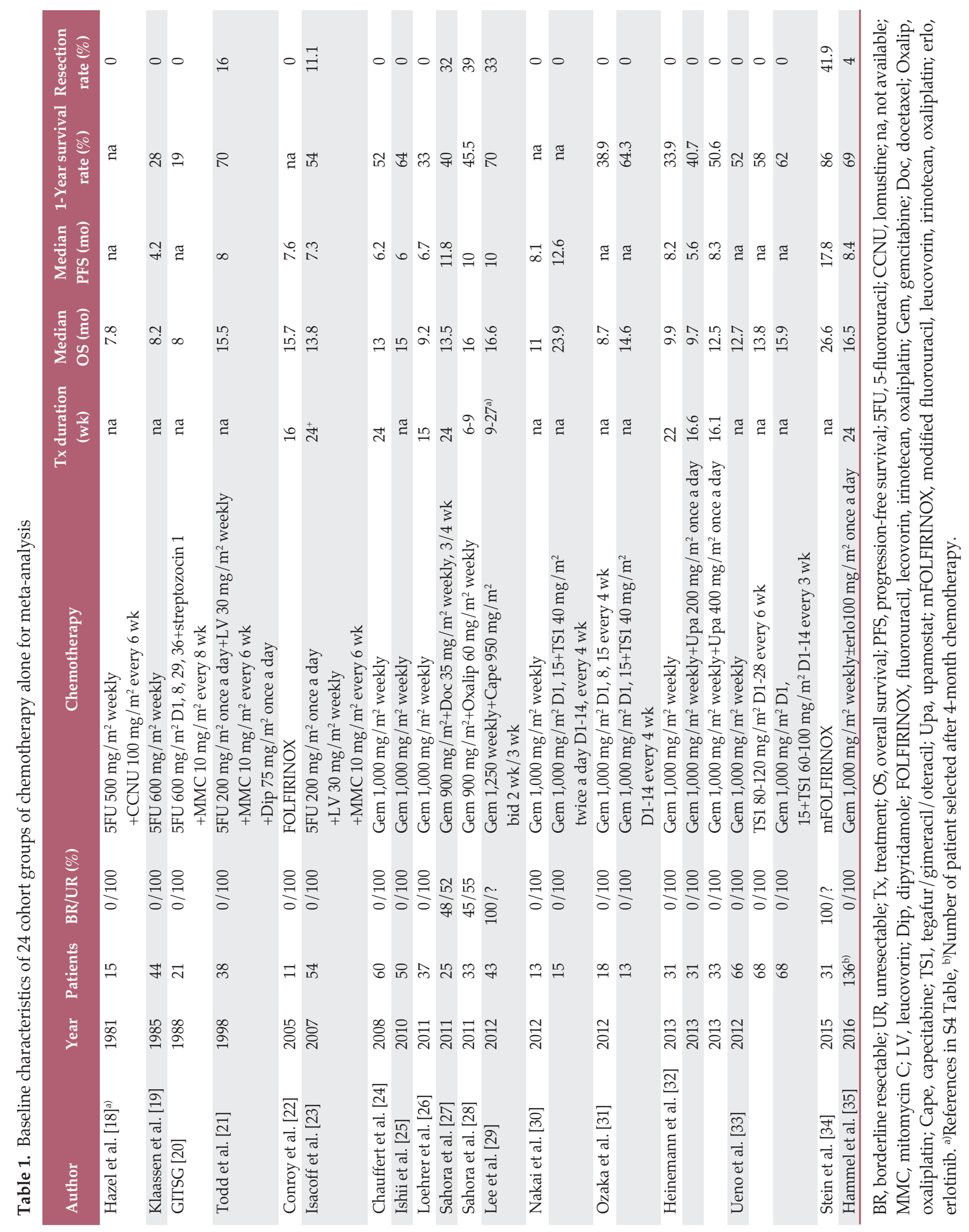




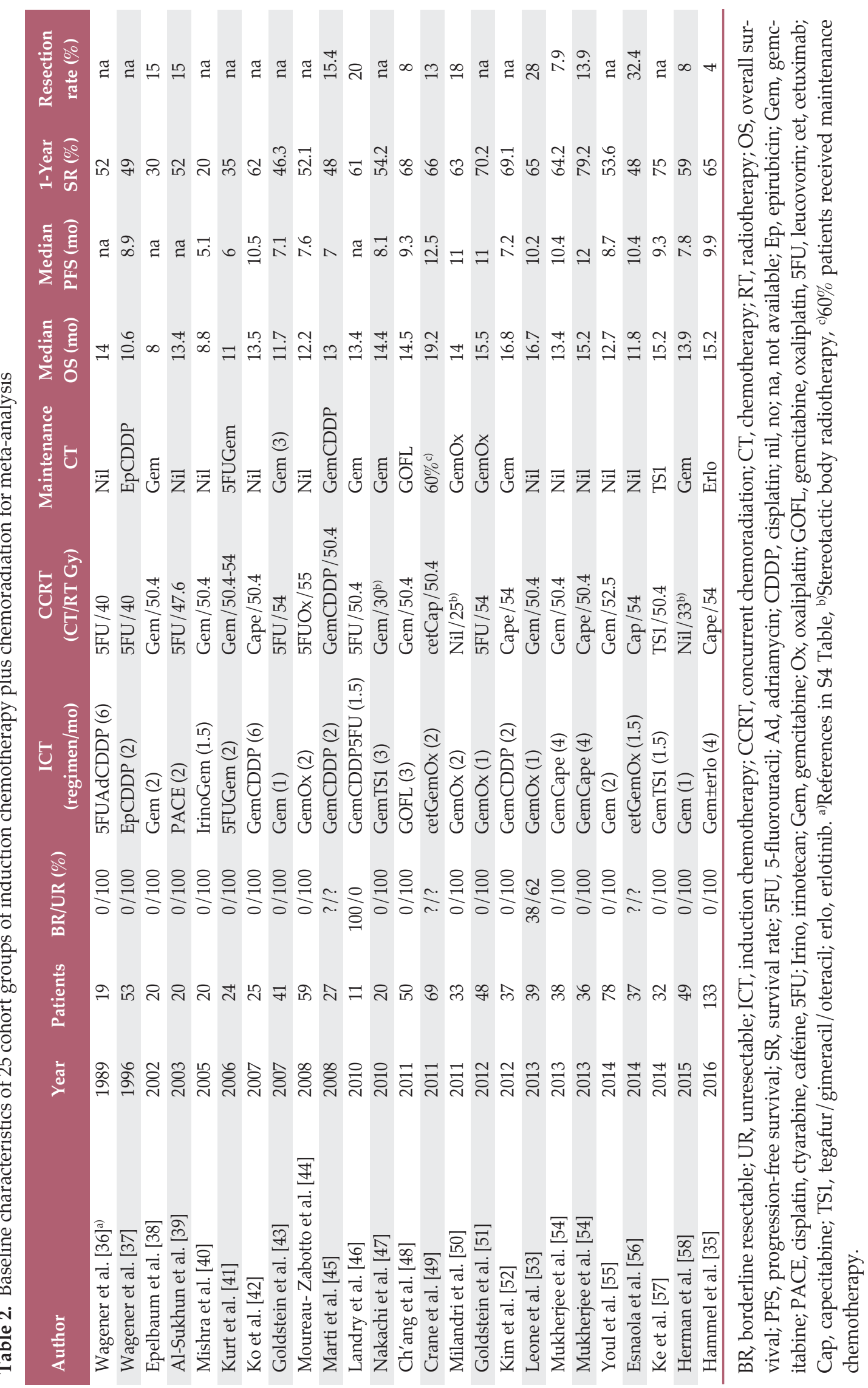




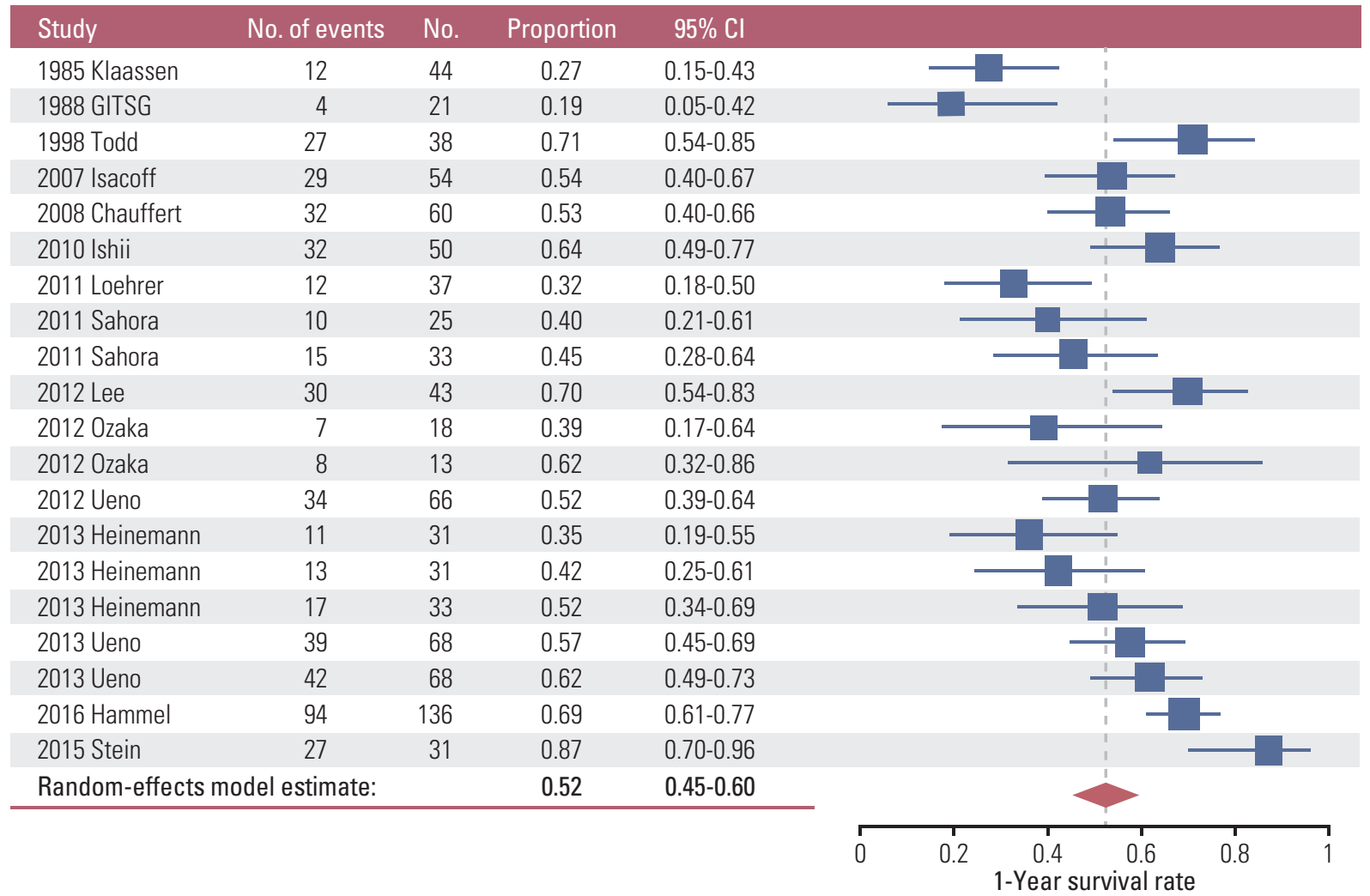

Fig. 2. Forest plot of 1-year survival [19-21,23-29,31-58]. (A) Chemotherapy groups. (B) ICT+CCRT groups. CI, confidence interval; ICT, induction chemotherapy; CCRT, chemoradiation. (Continued to the next page)

stereotactic body radiotherapy were without concomitant chemotherapy.

The meta-analysis showed that 1-year survival rate was not significantly different between chemotherapy alone (52\%; 95\% CI, 45 to 60) and ICT+CCRT (58\%; 95\% CI, 53 to 62) (Fig. 2). Funnel plots disclosed significant publication bias of both groups of studies (S10 Fig.). Studies using ICT+CCRT had a significantly better 1-year survival rate than chemotherapy alone when the duration of ICT was 3 months or more $(65 \%$; $95 \%$ CI, 60 to 70 vs. $52 \%$; $95 \%$ CI, 45 to 60) (Table 3). In randomized trials, a significantly better survival rate of the four ICT+CCRT studies was noted compared to that of the thirteen chemotherapy alone studies $(67 \%$; 95\% CI, 60 to 73 vs. $47 \%$; 95\% CI, 38 to 56) (Table 3). Similar observations were noted in meta-analysis of median OS or median PFS as end points with less statistical significance (S11 Table). For both groups, smaller studies showed bias toward poorer survival. Of the 14 and 13 studies in chemotherapy alone and ICT+CCRT groups, respectively, published after year 2010, there was a trend of survival benefit in ICT+CCRT over chemotherapy alone groups $(64 \%$; $95 \%$ CI, 60 to 68 vs. $55 \%$; 95\% CI, 47 to 61 ).

Combination chemotherapy as induction therapy did not potentiate the benefit of consolidation CCRT compared to those using single agent ( $58 \%$; 95\% CI, 52 to 64 vs. $58 \% ; 95 \%$ CI, 45 to 70) (Table 3). Maintenance chemotherapy after ICT+CCRT did not significantly affect the 1-year survival rate of LAPC compared to those with chemotherapy alone (59\%; $95 \%$ CI, 52 to 65 vs. 52\%; 95\% CI, 45 to 60). However, in the 10 ICT+CCRT study arms using maintenance chemotherapy published after 2010, there appeared to be survival benefit compared to those 15 study arms using chemotherapy alone (66\%; 95\% CI, 62 to 70 vs. 55\%; 95\% CI, 47 to 61$)$. The benefit of additional CCRT was not correlated with radiation dosage and the regimen of radiation sensitizer. However, a trend of survival benefit regarding ICT+CCRT over chemotherapy alone was found for using fluorouracil as radiosensitizer especially in studies published after 2010 (66\%; 95\% CI, 62 to 71 vs. 55\%; 95\% CI, 47 to 61 ).

Nine studies enrolling various proportion of patients with 


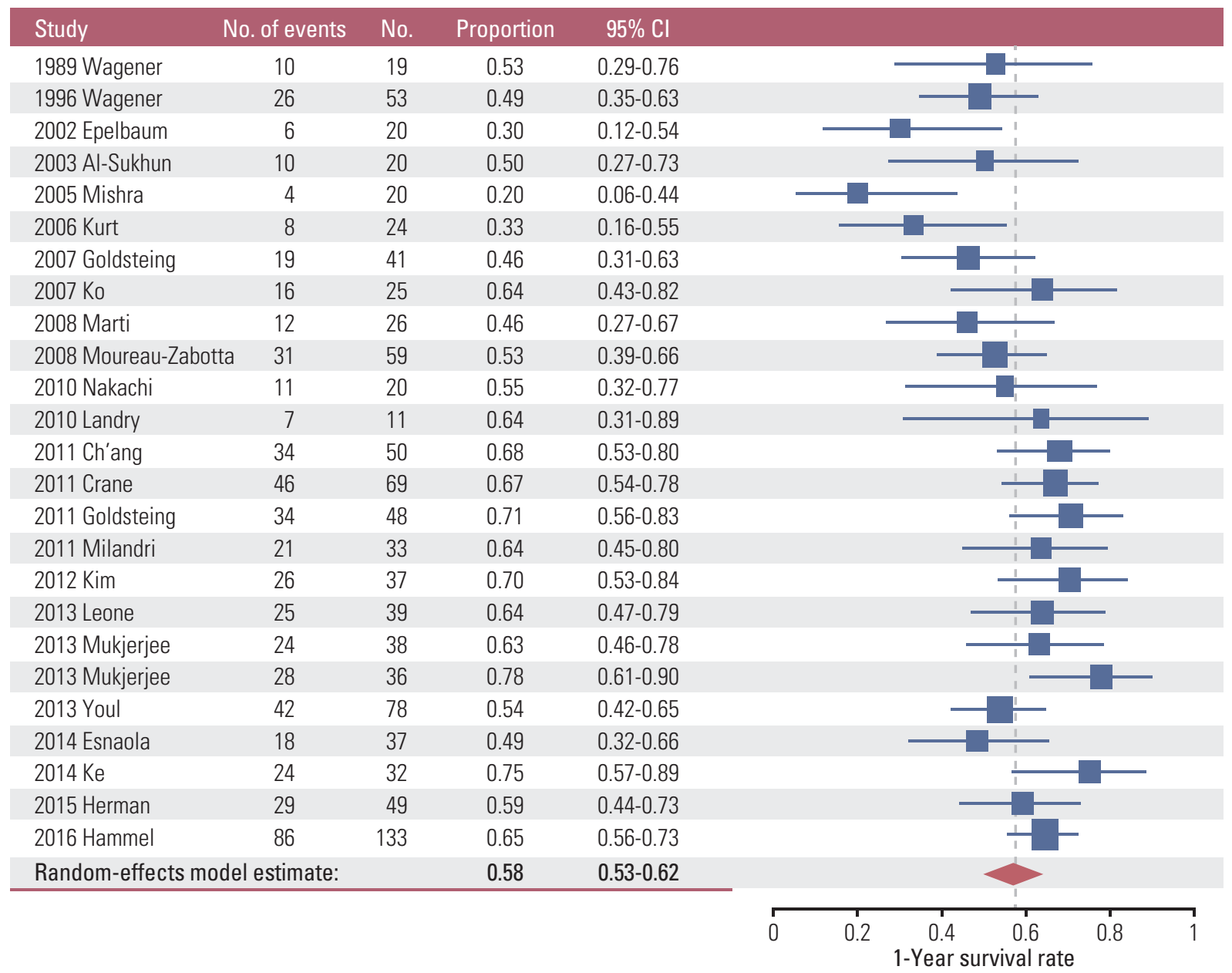

Fig. 2. (Continued from the previous page)

borderline disease had a trend of better survival. The survival benefit of ICT+CCRT over chemotherapy was not observed in studies enrolling unresectable disease alone or both borderline and unresectable pancreatic cancer (Table 3). Twenty studies reported resection after chemotherapy with or without chemoradiation. There was no significant correlation between resection and the use of CCRT (ICT+CCRT, 13\%; 95\% CI, 9 to 18 vs. chemotherapy alone, $23 \%$; $95 \%$ CI, 11 to 34$)$.

Seven of the $24 \mathrm{ICT}+\mathrm{CCRT}$ studies $(29.2 \%)$ and nine of the 22 chemotherapy alone studies $(40.9 \%)$ reported grade $3 / 4$ toxicity rate of more than $30 \%$. The development of hematologic (19\%; 95\% CI, 14 to 24 vs. $23 \%$; 95\% CI, 16 to 30$)$ and non-hematologic toxicity (19\%; $95 \%$ CI, 14 to 24 vs. $15 \%$; $95 \%$ CI, 11 to 20) was not significantly different between ICT+ CCRT and chemotherapy alone.

\section{Discussion}

The latest meta-analysis of randomized trials demonstrated no survival benefit of upfront chemoradiotherapy followed by chemotherapy versus chemotherapy alone [6]. Recent prospective clinical trials evaluating the role of CCRT after ICT in LAPC patients still revealed no survival benefit over chemotherapy alone [9]. Investigators suggested the incorporation of more active combination chemotherapy as induction therapy and modern radiotherapy techniques into the design of future clinical trials. To address the issue and provide further information, we conducted this systemic review and meta-analysis.

We found that there was no survival benefit of CCRT after ICT for LAPC from meta-analysis of 41 selected prospective clinical trials. The lack of survival benefit of CCRT in addi- 
Table 3. Comparison of 1-year survival between ICT+CCRT vs. CT

\begin{tabular}{|c|c|c|c|}
\hline Treatment & No. of studies & Meta-1-year survival (95\% CI) & $\mathrm{I}^{2}$ \\
\hline All & 45 & $0.55(0.51-0.59)$ & 70.6 \\
\hline CT & 20 & $0.52(0.45-0.60)$ & 78.3 \\
\hline ICT+CCRT & 25 & $0.58(0.53-0.62)$ & 59.7 \\
\hline \multicolumn{4}{|l|}{ Randomization } \\
\hline \multicolumn{4}{|l|}{ No } \\
\hline $\mathrm{CT}$ & 6 & $0.65(0.53-0.76)$ & 73.9 \\
\hline ICT+CCRT & 21 & $0.55(0.50-0.61)$ & 59.7 \\
\hline \multicolumn{4}{|l|}{ Yes } \\
\hline $\mathrm{CT}$ & 13 & $0.47(0.38-0.56)$ & 78.1 \\
\hline $\mathrm{ICT}+\mathrm{CCRT}^{*}$ & 4 & $\left.0.67(0.60-0.73)^{\star}, \mathrm{a}\right)$ & 0 \\
\hline \multicolumn{4}{|l|}{ Resectability status } \\
\hline \multicolumn{4}{|l|}{ Unresectable only } \\
\hline $\mathrm{CT}$ & 16 & $0.50(0.42-0.58)$ & 76.1 \\
\hline $\mathrm{ICT}+\mathrm{CCRT}$ & 20 & $0.57(0.51-0.63)$ & 64.8 \\
\hline \multicolumn{4}{|l|}{ Borderline and unresectable } \\
\hline $\mathrm{CT}$ & 4 & $0.62(0.40-0.82)$ & 85.4 \\
\hline $\mathrm{ICT}+\mathrm{CCRT}$ & 5 & $\left.0.59(0.52-0.66)^{a}\right)$ & 28.4 \\
\hline \multicolumn{4}{|l|}{ No. of chemotherapy agents } \\
\hline \multicolumn{4}{|l|}{ One } \\
\hline $\mathrm{CT}$ & 11 & $0.48(0.40-0.57)$ & 77.2 \\
\hline $\mathrm{ICT}+\mathrm{CCRT}$ & 6 & $0.56(0.47-0.65)$ & 64.4 \\
\hline \multicolumn{4}{|l|}{ Two or more } \\
\hline $\mathrm{CT}$ & 9 & $0.58(0.45-0.70)$ & 80.0 \\
\hline $\mathrm{ICT}+\mathrm{CCRT}$ & 19 & $0.58(0.52-0.64)$ & 60.5 \\
\hline \multicolumn{4}{|l|}{ ICT duration } \\
\hline $\mathrm{CT}$ & 20 & $0.52(0.45-0.60)$ & 78.3 \\
\hline $\mathrm{ICT}+\mathrm{CCRT}$ with $\mathrm{ICT}<3$ mo & 18 & $0.55(0.48-0.61)$ & 63.2 \\
\hline $\mathrm{ICT}+\mathrm{CCRT}$ with ICT $\geq 3 \mathrm{mo}^{*}$ & 7 & $\left.0.65(0.60-0.70)^{*}, \mathrm{a}\right)$ & 0 \\
\hline \multicolumn{4}{|l|}{ Maintenance CT } \\
\hline $\mathrm{CT}$ & 20 & $0.52(0.45-0.60)$ & 78.3 \\
\hline $\mathrm{ICT}+\mathrm{CCRT}$ without maintenance CT & 10 & $0.56(0.47-0.64)$ & 62.1 \\
\hline ICT+CCRT with maintenance CT & 15 & $0.59(0.52-0.65)$ & 59.0 \\
\hline \multicolumn{4}{|l|}{ RT dosage } \\
\hline CT & 20 & $0.52(0.45-0.60)$ & 78.3 \\
\hline ICT+CCRT with RT dosage < 50 Gy & 5 & $0.56(0.48-0.63)^{a)}$ & 0 \\
\hline ICT+CCRT with RT dosage $\geq 50$ Gy & 20 & $0.58(0.52-0.64)$ & 66.5 \\
\hline \multicolumn{4}{|l|}{ Radiosensitizer } \\
\hline CT & 20 & $0.52(0.45-0.60)$ & 78.3 \\
\hline ICT+CCRT with gemcitabine & 8 & $0.50(0.38-0.61)$ & 73.7 \\
\hline ICT+CCRT with fluorouracil & 12 & $\left.0.64(0.60-0.68)^{a}\right)$ & 40.7 \\
\hline ICT+CCRT with other & 3 & $0.56(0.48-0.65)^{\mathrm{a})}$ & 1.3 \\
\hline \multicolumn{4}{|l|}{ Study year } \\
\hline \multicolumn{4}{|l|}{ Before 2010} \\
\hline $\mathrm{CT}$ & 5 & $0.45(0.28-0.62)$ & 84.0 \\
\hline ICT+CCRT & 10 & $0.46(0.40-0.51)^{\mathrm{a})}$ & 40.6 \\
\hline \multicolumn{4}{|l|}{2010 or after 2010} \\
\hline $\mathrm{CT}$ & 15 & $0.55(0.47-0.62)$ & 74.3 \\
\hline $\mathrm{ICT}+\mathrm{CCRT}$ & 15 & $\left.0.64(0.60-0.68)^{a}\right)$ & 9.9 \\
\hline
\end{tabular}

ICT, induction chemotherapy; CCRT, chemoradiation; CT, chemotherapy; CI, confidence interval; RT, radiotherapy. * $<<0.05$. a)Based-on fixed-effects models. 
tion to chemotherapy was probably attributed to reasons including short duration of ICT, non-effective ICT regimen, non-optimal radiosensitizer, no maintenance chemotherapy in most clinical trials.

Subgroup analysis showed significant survival benefit of ICT+CCRT over chemotherapy alone when the duration of ICT lasted for at least three months. Consistent with our observation, a retrospective study from Johns Hopkins Hospital also demonstrated a significantly decreased cumulative incidence of progression $(p=0.008)$ and a trend toward better OS (19.4 months vs. 15.7 months, $\mathrm{p}=0.10)$ in patients who received $>2$ cycles of chemotherapy before CCRT in LAPC [59].

The role of maintenance chemotherapy following ICT + CCRT of LAPC is unknown. The CAIRO3 study evaluated the benefit of maintenance chemotherapy in metastatic colorectal cancer and found a significant improvement of PFS (11.7 months vs. 8.5 months, $\mathrm{p}<0.001)$ [60]. A retrospective study in pancreatic cancer also demonstrated the survival benefit of maintenance capecitabine after adjuvant treatment compared to adjuvant treatment alone with OS of 48.4 months vs 22.0 months $(p<0.001)$ [61]. In this study, a trend of survival benefit of additional CCRT was noted by the 27 clinical trials published after year 2010 that provided maintenance chemotherapy.

Previous randomized trials revealed that the combination regimen of FOLFIRINOX (fluorouracil, leucovorin, irinotecan, oxaliplatin) and gemcitabine plus nab-paclitaxel improved the OS of advanced pancreatic cancer significantly compared to that treated with gemcitabine alone $[62,63]$. In this analysis, we did not find the superiority of combination chemotherapy compared to single agent in chemotherapy alone studies, nor did we find the benefit of CCRT after ICT using combination regimen for LAPC patients. This result might be attributed to the lack of effective combination regimen in the 41 studies enrolled for meta-analysis. The ICT regimen most often used in the 25 study arms of our metaanalysis was gemcitabine with platinum analog which failed to improve survival over gemcitabine alone in several phase III trials in advanced pancreatic cancer $[64,65]$.

We found that ICT followed by 5 -fluorouracil (5FU)-based CCRT showed a trend of better survival compared to chemotherapy alone. A recent randomized trial and a metaanalysis suggested a superiority for $5 \mathrm{FU}$-based chemoradiotherapy over gemcitabine [66,67]. Investigators demonstrated that TS-1 or capecitabine had similar efficacy compared to gemcitabine as radiosensitizers, although TS- 1 and capecitabine were associated with fewer toxicities.

Our study has several limitations. The studies included in our meta-analysis were conducted at different times and resulted in heterogeneity in study subjects and treatment. The improvements in diagnostic imaging resulted in more accurate clinical staging of pancreatic cancer. The definition of LAPC had only been standardized recently. Some patients are now being reclassified as borderline resectable due to improvements in surgical techniques and perioperative care [68]. Besides, the various radiation dosages, target volumes, and techniques as well as different chemotherapy types and dosages administered might have affected the study results. Finally, potential publication bias exists for both the chemotherapy alone and ICT+CCRT groups, with smaller studies tended to show poorer survival. It is unclear how this publication bias might have affected our results; however, since the publication bias was in the same direction for both treatment groups, the overall impact should have been minimal.

Consensus guidelines of radiotherapy in LAPC have been developed to guide the desired doses and treatment volume [69]. Gemcitabine/nab-paclitaxel combination and FOLFIRINOX have been shown to improve disease control compared to gemcitabine alone in advanced pancreatic cancer. A recent randomized trial suggested a superiority of capecitabine as radiosensitizer over gemcitabine [66]. Investigator should consider the adoption of more active chemotherapy regimens as induction therapy and the incorporation of maintenance chemotherapy. The value of high-dose ablative stereotactic body radiotherapy should also be evaluated [70]. The development of biomarkers such as DPC4, Runx3 [1,71], and radiation sensitivity analyses [72] to select patients at high risk of developing local destructive disease for CCRT treatment is warranted.

In summary, the role of CCRT after ICT in LAPC patients is evolving. Our meta-analysis revealed no survival benefit of CCRT in addition to chemotherapy for LAPC patients. However, significant survival benefit of ICT+CCRT over chemotherapy alone was noted when ICT lasted for at least 3 months. A trend of clinical benefit using 5FU-based CCRT, and maintenance chemotherapy were also noted for LAPC.

\section{Electronic Supplementary Material}

Supplementary materials are available at Cancer Research and Treatment website (http:// www.e-crt.org).

\section{Conflicts of Interest}

Conflict of interest relevant to this article was not reported.

\section{Acknowledgments}

This work was supported by grants from National Health Research Institutes, Taiwan (CA-104-PP28 and in part by PH-104PP-04). 


\section{References}

1. Iacobuzio-Donahue CA, Fu B, Yachida S, Luo M, Abe H, Henderson CM, et al. DPC4 gene status of the primary carcinoma correlates with patterns of failure in patients with pancreatic cancer. J Clin Oncol. 2009;27:1806-13.

2. Moertel CG, Frytak S, Hahn RG, O'Connell MJ, Reitemeier RJ, Rubin J, et al. Therapy of locally unresectable pancreatic carcinoma: a randomized comparison of high dose (6000 rads) radiation alone, moderate dose radiation (4000 rads+5-fluorouracil), and high dose radiation+5-fluorouracil: The Gastrointestinal Tumor Study Group. Cancer. 1981;48:1705-10.

3. Burris HA 3rd, Moore MJ, Andersen J, Green MR, Rothenberg ML, Modiano MR, et al. Improvements in survival and clinical benefit with gemcitabine as first-line therapy for patients with advanced pancreas cancer: a randomized trial. J Clin Oncol. 1997; 15:2403-13.

4. Chauffert B, Mornex F, Bonnetain F, Rougier P, Mariette C, Bouche $\mathrm{O}$, et al. Phase III trial comparing intensive induction chemoradiotherapy (60 Gy, infusional 5-FU and intermittent cisplatin) followed by maintenance gemcitabine with gemcitabine alone for locally advanced unresectable pancreatic cancer. Definitive results of the 2000-01 FFCD/SFRO study. Ann Oncol. 2008;19:1592-9.

5. Loehrer PJ Sr, Feng Y, Cardenes H, Wagner L, Brell JM, Cella $\mathrm{D}$, et al. Gemcitabine alone versus gemcitabine plus radiotherapy in patients with locally advanced pancreatic cancer: an Eastern Cooperative Oncology Group trial. J Clin Oncol. 2011;29:4105-12.

6. Sultana A, Tudur Smith C, Cunningham D, Starling N, Tait D, Neoptolemos JP, et al. Systematic review, including metaanalyses, on the management of locally advanced pancreatic cancer using radiation/combined modality therapy. Br J Cancer. 2007;96:1183-90.

7. Huguet F, Andre T, Hammel P, Artru P, Balosso J, Selle F, et al. Impact of chemoradiotherapy after disease control with chemotherapy in locally advanced pancreatic adenocarcinoma in GERCOR phase II and III studies. J Clin Oncol. 2007;25: 326-31.

8. Krishnan S, Rana V, Janjan NA, Varadhachary GR, Abbruzzese JL, Das P, et al. Induction chemotherapy selects patients with locally advanced, unresectable pancreatic cancer for optimal benefit from consolidative chemoradiation therapy. Cancer. 2007;110:47-55.

9. Hammel P, Huguet F, van Laethem JL, Goldstein D, Glimelius B, Artru P, et al. Effect of chemoradiotherapy vs chemotherapy on survival in patients with locally advanced pancreatic cancer controlled after 4 months of gemcitabine with or without erlotinib: the LAP07 randomized clinical trial. JAMA. 2016;315:1844-53.

10. Abrams RA, Winter KA, Regine WF, Safran H, Hoffman JP, Lustig R, et al. Failure to adhere to protocol specified radiation therapy guidelines was associated with decreased survival in RTOG 9704: a phase III trial of adjuvant chemotherapy and chemoradiotherapy for patients with resected adenocarcinoma of the pancreas. Int J Radiat Oncol Biol Phys. 2012;82:
809-16.

11. Moher D, Liberati A, Tetzlaff J, Altman DG; PRISMA Group. Preferred reporting items for systematic reviews and metaanalyses: the PRISMA statement. PLoS Med. 2009;6:e1000097.

12. Schwarzer G. meta: an R package for meta-analysis. R News. 2007;7:40-5.

13. Freeman MF, Tukey JW. Transformations related to the angular and the square root. Ann Math Stat. 1950;21:607-11.

14. Higgins JP, Thompson SG, Deeks JJ, Altman DG. Measuring inconsistency in meta-analyses. BMJ. 2003;327:557-60.

15. Lau J, Ioannidis JP, Schmid CH. Quantitative synthesis in systematic reviews. Ann Intern Med. 1997;127:820-6.

16. Egger M, Davey Smith G, Schneider M, Minder C. Bias in meta-analysis detected by a simple, graphical test. BMJ. 1997;315:629-34.

17. Sterne JA, Egger M, Smith GD. Systematic reviews in health care: Investigating and dealing with publication and other biases in meta-analysis. BMJ. 2001;323:101-5.

18. Hazel JJ, Thirlwell MP, Huggins M, Maksymiuk A, MacFarlane JK. Multi-drug chemotherapy with and without radiation for carcinoma of the stomach and pancreas: a prospective randomized trial. J Can Assoc Radiol. 1981;32:164-5.

19. Klaassen DJ, MacIntyre JM, Catton GE, Engstrom PF, Moertel CG. Treatment of locally unresectable cancer of the stomach and pancreas: a randomized comparison of 5-fluorouracil alone with radiation plus concurrent and maintenance 5-fluorouracil: an Eastern Cooperative Oncology Group study. J Clin Oncol. 1985;3:373-8.

20. Treatment of locally unresectable carcinoma of the pancreas: comparison of combined-modality therapy (chemotherapy plus radiotherapy) to chemotherapy alone. Gastrointestinal Tumor Study Group. J Natl Cancer Inst. 1988;80:751-5.

21. Todd KE, Gloor B, Lane JS, Isacoff WH, Reber HA. Resection of locally advanced pancreatic cancer after downstaging with continuous-infusion 5-fluorouracil, mitomycin-C, leucovorin, and dipyridamole. J Gastrointest Surg. 1998;2:159-66.

22. Conroy T, Paillot B, Francois E, Bugat R, Jacob JH, Stein U, et al. Irinotecan plus oxaliplatin and leucovorin-modulated fluorouracil in advanced pancreatic cancer: a Groupe Tumeurs Digestives of the Federation Nationale des Centres de Lutte Contre le Cancer study. J Clin Oncol. 2005;23:1228-36.

23. Isacoff WH, Bendetti JK, Barstis JJ, Jazieh AR, Macdonald JS, Philip PA. Phase II trial of infusional fluorouracil, leucovorin, mitomycin, and dipyridamole in locally advanced unresectable pancreatic adenocarcinoma: SWOG S9700. J Clin Oncol. 2007;25:1665-9.

24. Chauffert B, Mornex F, Bonnetain F, Rougier P, Mariette C, Bouche $\mathrm{O}$, et al. Phase III trial comparing intensive induction chemoradiotherapy (60 Gy, infusional 5-FU and intermittent cisplatin) followed by maintenance gemcitabine with gemcitabine alone for locally advanced unresectable pancreatic cancer: definitive results of the 2000-01 FFCD/SFRO study. Ann Oncol. 2008;19:1592-9.

25. Ishii H, Furuse J, Boku N, Okusaka T, Ikeda M, Ohkawa S, et 
al. Phase II study of gemcitabine chemotherapy alone for locally advanced pancreatic carcinoma: JCOG0506. Jpn J Clin Oncol. 2010;40:573-9.

26. Loehrer PJ Sr, Feng Y, Cardenes H, Wagner L, Brell JM, Cella $\mathrm{D}$, et al. Gemcitabine alone versus gemcitabine plus radiotherapy in patients with locally advanced pancreatic cancer: an Eastern Cooperative Oncology Group trial. J Clin Oncol. 2011;29:4105-12.

27. Sahora K, Kuehrer I, Schindl M, Koelblinger C, Goetzinger P, Gnant M. NeoGemTax: gemcitabine and docetaxel as neoadjuvant treatment for locally advanced nonmetastasized pancreatic cancer. World J Surg. 2011;35:1580-9.

28. Sahora K, Kuehrer I, Eisenhut A, Akan B, Koellblinger C, Goetzinger $\mathrm{P}$, et al. NeoGemOx: gemcitabine and oxaliplatin as neoadjuvant treatment for locally advanced, nonmetastasized pancreatic cancer. Surgery. 2011;149:311-20.

29. Lee JL, Kim SC, Kim JH, Lee SS, Kim TW, Park DH, et al. Prospective efficacy and safety study of neoadjuvant gemcitabine with capecitabine combination chemotherapy for borderline-resectable or unresectable locally advanced pancreatic adenocarcinoma. Surgery. 2012;152:851-62.

30. Nakai $Y$, Isayama $H$, Sasaki T, Sasahira N, Tsujino T, Toda N, et al. A multicentre randomised phase II trial of gemcitabine alone vs gemcitabine and S-1 combination therapy in advanced pancreatic cancer: GEMSAP study. Br J Cancer. 2012;106:1934-9.

31. Ozaka M, Matsumura Y, Ishii H, Omuro Y, Itoi T, Mouri H, et al. Randomized phase II study of gemcitabine and S-1 combination versus gemcitabine alone in the treatment of unresectable advanced pancreatic cancer (Japan Clinical Cancer Research Organization PC-01 study). Cancer Chemother Pharmacol. 2012;69:1197-204.

32. Heinemann V, Ebert MP, Laubender RP, Bevan P, Mala C, Boeck S. Phase II randomised proof-of-concept study of the urokinase inhibitor upamostat (WX-671) in combination with gemcitabine compared with gemcitabine alone in patients with non-resectable, locally advanced pancreatic cancer. Br J Cancer. 2013;108:766-70.

33. Ueno M, Okusaka T, Mizusawa J, Takashima A, Morizane C, Ikeda M, et al. Randomized phase II trial of gemcitabine plus S-1 combination therapy versus S-1 in advanced biliary tract cancer: results of the Japan Clinical Oncology Group study (JCOG0805). J Clin Oncol. 2012;30(15 Suppl):4031.

34. Stein S, Cong X, Yao X, Hahn C, Li J, Kortmansky JS, et al. Phase II study of Yale modified FOLFIRINOX (mFOLFIRINOX) in locally advanced pancreatic cancer (LAPC). J Clin Oncol. 2015;33(15 Suppl):e15274.

35. Hammel P, Huguet F, van Laethem JL, Goldstein D, Glimelius B, Artru P, et al. Effect of chemoradiotherapy vs chemotherapy on survival in patients with locally advanced pancreatic cancer controlled after 4 months of gemcitabine with or without erlotinib: the LAP07 randomized clinical trial. JAMA. 2016; 315:1844-53.

36. Wagener DJ, van Hoesel QG, Yap SH, Hoogenraad WJ, Wobbes T, Strijk SP. Phase II trial of 5-fluorouracil, adriamycin and cisplatin (FAP) followed by radiation and 5-fluorouracil in locally advanced pancreatic cancer. Cancer Chemother Pharmacol. 1989;25:131-4.

37. Wagener DJ, Hoogenraad WJ, Rougier P, Lusinchi A, Taal BG, Veenhof $\mathrm{CH}$, et al. Results of a phase II trial of epirubicin and cisplatin (EP) before and after irradiation and 5-fluorouracil in locally advanced pancreatic cancer: an EORTC GITCCG study. Eur J Cancer. 1996;32A:1310-3.

38. Epelbaum R, Rosenblatt E, Nasrallah S, Faraggi D, Gaitini D, Mizrahi S, et al. Phase II study of gemcitabine combined with radiation therapy in patients with localized, unresectable pancreatic cancer. J Surg Oncol. 2002;81:138-43.

39. Al-Sukhun S, Zalupski MM, Ben-Josef E, Vaitkevicius VK, Philip PA, Soulen R, et al. Chemoradiotherapy in the treatment of regional pancreatic carcinoma: a phase II study. Am J Clin Oncol. 2003;26:543-9.

40. Mishra G, Butler J, Ho C, Melin S, Case LD, Ennever PR, et al. Phase II trial of induction gemcitabine/CPT-11 followed by a twice-weekly infusion of gemcitabine and concurrent external beam radiation for the treatment of locally advanced pancreatic cancer. Am J Clin Oncol. 2005;28:345-50.

41. Kurt E, Kurt M, Kanat O, Cetintas SK, Aygun S, Palazoglu T, et al. Phase II study of induction chemotherapy with gemcitabine plus 5 -fluorouracil followed by gemcitabine-based concurrent chemoradiotherapy for unresectable locally advanced pancreatic cancer. Tumori. 2006;92:481-6.

42. Ko AH, Quivey JM, Venook AP, Bergsland EK, Dito E, Schillinger B, et al. A phase II study of fixed-dose rate gemcitabine plus low-dose cisplatin followed by consolidative chemoradiation for locally advanced pancreatic cancer. Int J Radiat Oncol Biol Phys. 2007;68:809-16.

43. Goldstein D, Van Hazel G, Walpole E, Underhill C, Kotasek D, Michael M, et al. Gemcitabine with a specific conformal 3D 5FU radiochemotherapy technique is safe and effective in the definitive management of locally advanced pancreatic cancer. Br J Cancer. 2007;97:464-71.

44. Moureau-Zabotto L, Phelip JM, Afchain P, Mineur L, Andre $\mathrm{T}$, Vendrely V, et al. Concomitant administration of weekly oxaliplatin, fluorouracil continuous infusion, and radiotherapy after 2 months of gemcitabine and oxaliplatin induction in patients with locally advanced pancreatic cancer: a Groupe Coordinateur Multidisciplinaire en Oncologie phase II study. J Clin Oncol. 2008;26:1080-5.

45. Marti JL, Hochster HS, Hiotis SP, Donahue B, Ryan T, Newman E. Phase I/II trial of induction chemotherapy followed by concurrent chemoradiotherapy and surgery for locoregionally advanced pancreatic cancer. Ann Surg Oncol. 2008;15: 3521-31.

46. Landry J, Catalano PJ, Staley C, Harris W, Hoffman J, Talamonti M, et al. Randomized phase II study of gemcitabine plus radiotherapy versus gemcitabine, 5-fluorouracil, and cisplatin followed by radiotherapy and 5-fluorouracil for patients with locally advanced, potentially resectable pancreatic adenocarcinoma. J Surg Oncol. 2010;101:587-92.

47. Nakachi K, Furuse J, Kinoshita T, Kawashima M, Ishii H, Ikeda $\mathrm{M}$, et al. A phase II study of induction chemotherapy with gemcitabine plus S-1 followed by chemoradiotherapy for 
locally advanced pancreatic cancer. Cancer Chemother Pharmacol. 2010;66:527-34.

48. Ch'ang HJ, Lin YL, Wang HP, Chiu YF, Chang MC, Hsu CH, et al. Induction chemotherapy with gemcitabine, oxaliplatin, and 5-fluorouracil/leucovorin followed by concomitant chemoradiotherapy in patients with locally advanced pancreatic cancer: a Taiwan cooperative oncology group phase II study. Int J Radiat Oncol Biol Phys. 2011;81:e749-57.

49. Crane $\mathrm{CH}$, Varadhachary GR, Yordy JS, Staerkel GA, Javle MM, Safran H, et al. Phase II trial of cetuximab, gemcitabine, and oxaliplatin followed by chemoradiation with cetuximab for locally advanced (T4) pancreatic adenocarcinoma: correlation of Smad4(Dpc4) immunostaining with pattern of disease progression. J Clin Oncol. 2011;29:3037-43.

50. Milandri C, Polico R, Garcea D, Passardi A, Gardini A, Romeo A, et al. GEMOX plus tomotherapy for unresectable locally advanced pancreatic cancer. Hepatogastroenterology. 2011;58: 599-603.

51. Goldstein D, Spry N, Cummins MM, Brown C, van Hazel GA, Carroll S, et al. The GOFURTGO Study: AGITG phase II study of fixed dose rate gemcitabine-oxaliplatin integrated with concomitant 5FU and 3-D conformal radiotherapy for the treatment of localised pancreatic cancer. Br J Cancer. 2012;106:61-9.

52. Kim JS, Lim JH, Kim JH, Im SA, Chie EK, Hwang JH, et al. Phase II clinical trial of induction chemotherapy with fixed dose rate gemcitabine and cisplatin followed by concurrent chemoradiotherapy with capecitabine for locally advanced pancreatic cancer. Cancer Chemother Pharmacol. 2012;70: 381-9.

53. Leone F, Gatti M, Massucco P, Colombi F, Sperti E, Campanella $\mathrm{D}$, et al. Induction gemcitabine and oxaliplatin therapy followed by a twice-weekly infusion of gemcitabine and concurrent external-beam radiation for neoadjuvant treatment of locally advanced pancreatic cancer: a single institutional experience. Cancer. 2013;119:277-84.

54. Mukherjee S, Hurt C, Griffiths G, Bridgewater JA, Crosby T, Falk S, et al. SCALOP: results of a randomized phase II study of induction chemotherapy followed by gemcitabine $(G)$ or capecitabine (Cap) based chemoradiation (CRT) in locally advanced pancreatic cancer (LANPC). J Clin Oncol. 2013;31 (4 Suppl):LBA146.

55. Youl M, Hashem S, Brade A, Cummings B, Dawson LA, Gallinger S, et al. Induction gemcitabine plus concurrent gemcitabine and radiotherapy for locally advanced unresectable or resected pancreatic cancer. Clin Oncol (R Coll Radiol). 2014;26:203-9.

56. Esnaola NF, Chaudhary UB, O'Brien P, Garrett-Mayer E, Camp ER, Thomas MB, et al. Phase 2 trial of induction gemcitabine, oxaliplatin, and cetuximab followed by selective capecitabine-based chemoradiation in patients with borderline resectable or unresectable locally advanced pancreatic cancer. Int J Radiat Oncol Biol Phys. 2014;88:837-44.

57. Ke QH, Zhou SQ, Yang JY, Du W, Liang G, Lei Y, et al. S-1 plus gemcitabine chemotherapy followed by concurrent radiotherapy and maintenance therapy with S-1 for unresectable pancreatic cancer. World J Gastroenterol. 2014;20:
13987-92.

58. Herman JM, Chang DT, Goodman KA, Dholakia AS, Raman SP, Hacker-Prietz A, et al. Phase 2 multi-institutional trial evaluating gemcitabine and stereotactic body radiotherapy for patients with locally advanced unresectable pancreatic adenocarcinoma. Cancer. 2015;121:1128-37.

59. Faisal F, Tsai HL, Blackford A, Olino K, Xia C, De Jesus-Acosta $\mathrm{A}$, et al. Longer course of induction chemotherapy followed by chemoradiation favors better survival outcomes for patients with locally advanced pancreatic cancer. Am J Clin Oncol. 2016;39:18-26.

60. Simkens LH, van Tinteren H, May A, ten Tije AJ, Creemers GJ, Loosveld OJ, et al. Maintenance treatment with capecitabine and bevacizumab in metastatic colorectal cancer (CAIRO3): a phase 3 randomised controlled trial of the Dutch Colorectal Cancer Group. Lancet. 2015;385:1843-52.

61. Weinberg BA, Wang H, Yang X, Wu CS, Pishvaian MJ, He AR, et al. Maintenance therapy with capecitabine in patients with resected pancreatic adenocarcinoma after adjuvant therapy: a retrospective cohort study. Gastrointest Cancer Res. 2014;7: 91-7.

62. Conroy T, Desseigne F, Ychou M, Bouche O, Guimbaud R, Becouarn $\mathrm{Y}$, et al. FOLFIRINOX versus gemcitabine for metastatic pancreatic cancer. N Engl J Med. 2011;364:1817-25.

63. Von Hoff DD, Ervin T, Arena FP, Chiorean EG, Infante J, Moore $\mathrm{M}$, et al. Increased survival in pancreatic cancer with nab-paclitaxel plus gemcitabine. N Engl J Med. 2013;369:1691703.

64. Heinemann V, Quietzsch D, Gieseler F, Gonnermann M, Schonekas H, Rost A, et al. Randomized phase III trial of gemcitabine plus cisplatin compared with gemcitabine alone in advanced pancreatic cancer. J Clin Oncol. 2006;24:3946-52.

65. Louvet C, Labianca R, Hammel P, Lledo G, Zampino MG, Andre T, et al. Gemcitabine in combination with oxaliplatin compared with gemcitabine alone in locally advanced or metastatic pancreatic cancer: results of a GERCOR and GISCAD phase III trial. J Clin Oncol. 2005;23:3509-16.

66. Mukherjee S, Hurt CN, Bridgewater J, Falk S, Cummins S, Wasan $\mathrm{H}$, et al. Gemcitabine-based or capecitabine-based chemoradiotherapy for locally advanced pancreatic cancer (SCALOP): a multicentre, randomised, phase 2 trial. Lancet Oncol. 2013;14:317-26.

67. Yang YF, Cao XH, Bao CE, Wan X. Concurrent radiotherapy with oral fluoropyrimidine versus gemcitabine in locally advanced pancreatic cancer: a systematic review and metaanalysis. Onco Targets Ther. 2015;8:3315-22.

68. Vauthey JN, Dixon E. AHPBA/SSO/SSAT Consensus Conference on Resectable and Borderline Resectable Pancreatic Cancer: rationale and overview of the conference. Ann Surg Oncol. 2009;16:1725-6.

69. Huguet F, Goodman KA, Azria D, Racadot S, Abrams RA. Radiotherapy technical considerations in the management of locally advanced pancreatic cancer: American-French consensus recommendations. Int J Radiat Oncol Biol Phys. 2012;83: 1355-64.

70. Trakul N, Koong AC, Chang DT. Stereotactic body radiother- 
apy in the treatment of pancreatic cancer. Semin Radiat Oncol. 2014;24:140-7.

71. Whittle MC, Izeradjene K, Rani PG, Feng L, Carlson MA, DelGiorno KE, et al. RUNX3 controls a metastatic switch in pan- creatic ductal adenocarcinoma. Cell. 2015;161:1345-60.

72. Torres-Roca JF. A molecular assay of tumor radiosensitivity: a roadmap towards biology-based personalized radiation therapy. Per Med. 2012;9:547-57. 